\title{
NMR Studies of Molecular Motion and Compatibility in Polymer Blends of Poly(epichlorohydrin)/Poly(methyl methacrylate)
}

\author{
Atsuhiko Yamanaka, Atsushi KaJI, and Masao Murano* \\ Toyobo Research Institute, Toyobo Co., Ltd. Katata, Ohtsu, Shiga 520-02, Japan \\ * Faculty of Culture and Education, Saga University, 1, Honjomachi, Saga 840, Japan
}

(Received July 14, 1997)

\begin{abstract}
Molecular motion of polymer chains depends on blending and differs in bulk and in polymer blend. In this research the molecular motion of poly(epichlorohydrin) $(\mathrm{PECH})$ was studied by ${ }^{13} \mathrm{C} \mathrm{NMR}$ and showed different behavior in bulk from polymer blend. Two types of blend films, $\mathrm{PECH} /$ poly(methyl methacrylate) $(\mathrm{PMMA})=(60 / 40)(\mathrm{wt} \%)$, were prepared from toluene and tetrahydrofuran (THF) solution; the former is transparent and the latter is opaque film. The line shape and temperature dependence of $T_{1}$ and $T_{2}$ in $\mathrm{PECH}$ indicate that the molecular motion of $\mathrm{PECH}$ is inhibited by surrounding PMMA chains and that the decrease in molecular motion depends on miscibility. The slow motion described by $T_{2}$ is more influenced by blending than the fast one described by $T_{1}$. Distribution curves of $T_{2}$ were obtained for bulk and three types of blend films prepared from toluene, THF, and $\mathrm{THF} / \mathrm{H}_{2} \mathrm{O}$ solution. The bulk and blend films cast from toluene show sharp distributions are homogeneous in molecular motions. The blend films cast from THF and THF/ $\mathrm{H}_{2} \mathrm{O}$ have wide distribution in molecular motion, which indicates the existence of the gradient in local concentration. $T_{1}$ in PMMA agrees with $T_{1}$ and $T_{2}$ in $\mathrm{PECH}$.

KEY WORDS $\quad{ }^{13} \mathrm{C}$ Nuclear Magnetic Reasonance / Poly(methyl methacrylate)/ Poly(epichlorohydrin) / $T_{1} / T_{2} /$ Distribution / Polymer Blend / Phase Structure / Molecular Motion /
\end{abstract}

The blending of polymers offers the possibilities of tailoring new technically important materials with specified properties. ${ }^{1}$ Some physical properties of polymer blends depend on chemical composition and miscibilities. $^{2-5}$ Especially thermal properties, for example glass transition temperature $T_{\mathrm{g}}$, are studied on many kinds of polymer blends ${ }^{1,3-5}$ and show that molecular motions of polymer chains are different in bulk and in blend.

NMR is one of the suitable methods to study molecular motions of polymer chain. ${ }^{6}$ There have been a lot of studies on the compatibilities of polymer blends by relaxation behavior of pulse NMR. ${ }^{7-10}$ High resolution NMR shows molecular mobilities in line shape and in relaxation time. ${ }^{6,11,12}$ Recently there are many studies on molecular motions of polymer chain at solid state in relation to relaxation time of solid state high resolution NMR. ${ }^{13,14}$ Relaxation time and spin-diffusion are applied to studies on morphology of inhomogeneous polymer system, such as crystalline/amorphous ${ }^{13,15}$ or polymer blend. ${ }^{13,18}$ Especially spin-lattice relaxation time of ${ }^{1} \mathrm{H}$ and spin diffusion have been applied to many studies on polymer blends. ${ }^{18-21}$ Above the glass transition temperature $T_{\mathrm{g}},{ }^{13} \mathrm{C}$ high resolution NMR spectra are obtainable for solid polymer even by conventional NMR spectrometers used for liquids. Molecular mobilities of solid polyesters above $T_{\mathrm{g}}$ were studied by $\mathrm{F}$. Horii et al. in this method. ${ }^{22}$

Poly(epichlorohydrin) (PECH) is a rubber like polymer and poly(methyl methacrylate) (PMMA) is a glassy polymer at room temperature. Their structures are shown in Figure 1. PECH is miscible with $\mathrm{PMMA}^{23}$ and this mixture shows low critical solution temperature (LCST) behaviors by DSC. ${ }^{24}$

But the molecular behavior of $\mathrm{PECH}$ polymer chain in blend film is unknown. This work studies the differences of molecular motion of $\mathrm{PECH}$ polymer chain for two blend films (transparent film and opaque film) with PMMA and bulk by NMR. We observed the behavior of PMMA polymer chain by solid state ${ }^{13} \mathrm{C}$ NMR. In the case of opaque film we observed the behavior of $\mathrm{PECH}$ polymer chain about the blend cast from tetrahydrofuran (THF) $/ \mathrm{H}_{2} \mathrm{O}$ to know the influence of compatibility by blending poor soluvent in cast solvent.

\section{EXPERIMENTAL}

\section{Sample Preparation}

Blended PECH/PMMA was prepared by solvent casting. Three PECH/PMMA = 60/40 (wt \%) blend films were prepared on petri dishes from toluene, THF, and $\mathrm{THF} / \mathrm{H}_{2} \mathrm{O}=90 / 10(\mathrm{wt} \%$ ) solution containing $2 \mathrm{wt} \%$ polymer. All films were dried for $24 \mathrm{~h}$ at $80^{\circ} \mathrm{C}$ under vacuum. Table I shows the appearance and $T_{\mathrm{g}}$ of the samples. Transparent blend film shows they are miscible and opaque films show phase separated.

\section{NMR Measurements}

${ }^{13} \mathrm{C}$ NMR spectra were measured using Varian XL-300 $\left({ }^{13} \mathrm{C} ; 75.5 \mathrm{MHz}\right)$ by conventional NMR spectrometers used for liquids. We measured temperature dependence

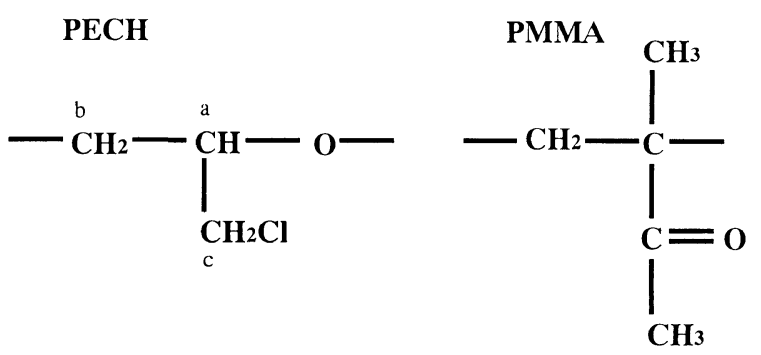

Figure 1. Structures of poly(epichlorohydrin) (PECH) and poly(methyl methacrylate) (PMMA). 
Table I. $T_{\mathrm{g}}$ and appearance of blend film

\begin{tabular}{cccc}
\hline Sample & Cast solvent & Appearance & $T_{\mathrm{g}} /{ }^{\circ} \mathrm{C}$ \\
\hline Blend 1 & Toluene & Transparent & 14 \\
Blend 2 & THF & Opaque & $-11,38$ \\
Blend 3 & THF $/ \mathrm{H}_{2} \mathrm{O}=90 / 10$ & Opaque & $-20,66$ \\
\hline
\end{tabular}

$T_{\mathrm{g}}(\mathrm{PECH})=-28.5^{\circ} \mathrm{C} ; T_{\mathrm{g}}(\mathrm{PMMA})=116.0^{\circ} \mathrm{C}$.

of spin-lattice relaxation time $\left(T_{1}\right)$ and spin-spin relaxation time $\left(T_{2}\right)$ for the bulk of $\mathrm{PECH}$, the blend film cast from toluene, and that cast from THF solution. Temperature was increased from $40^{\circ} \mathrm{C}$ to $140^{\circ} \mathrm{C}$. $T_{1}$ was measured by inversion recovery method and $T_{2}$ by Carr-Purcell-Meiboom-Gill method. ${ }^{6}$ The dimensions of samples were $50 \mathrm{~mm} \times 5 \mathrm{~mm} \times 1 \mathrm{~mm}$. For the blend film cast from $\mathrm{THF} / \mathrm{H}_{2} \mathrm{O}$, we measured only $T_{2}$ at $140^{\circ} \mathrm{C}$.

We measured $T_{1}$ by solid state high resolution ${ }^{13} \mathrm{C}$ NMR spectra to observe the molecular behavior of PMMA polymer chain in bulk and blend films. Solid state high resolution ${ }^{13} \mathrm{C}$ NMR spectra were measured by high power proton decoupling (DD) and magic angle spinning (MAS). Pulse width was $5 \mu$ s. Dipole decoupling power was about $50 \mathrm{kHz}$. $T_{1}$ was measured by inversion recovery method. The magic angle sample spinning rete was about $3.5 \mathrm{kHz}$.

\section{RESULTS AND DISCUSSION}

\section{${ }^{13}$ C NMR Spectra}

Figure 2 shows the ${ }^{13} \mathrm{C}$ NMR spectra of $\mathrm{PECH}$ in the bulk, blend film cast from toluene, and that cast from $\mathrm{THF}$ at $80^{\circ} \mathrm{C}$. The spectra have three peaks of methine, methylene, and chloromethylene carbon peaks in $\mathrm{PECH}$. The peaks in PMMA are not observed even above $T_{\mathrm{g}}$ which suggests that molecular motion of PMMA in blend films is too small for appearance of the spectra. The peaks of blends are broader than those of bulk. The peaks of transparent blend (cast from toluene) are broader than those of opaque film.

PECH peaks are broadened by blending which suggests that the molecular motion of $\mathrm{PECH}$ is hindered by PMMA polymer chain nearby $\mathrm{PECH}$.

Temperature Dependence of Spin-Lattice Relaxation Time $\left(T_{1}\right)$ in $\mathrm{PECH}$

Temperature dependence of $T_{1}$ in methine carbon is shown in Figure 3. As shown in Figure $3 T_{1}$ of methine carbon decreases, passing through a minimum and then increases with increasing temperature. But the temperature $\left(T_{\min }\right)$ where $T_{1}$ shows a minimum value in Table II is different for the samples as shown in Figure 3. $T_{\min }$ shifts to higher temperature by blending; $T_{\min }$ (bulk) $<$ $T_{\min }$ (opaque blend film cast from $\mathrm{THF}$ ) $<T_{\min }$ (transparent blend film cast from toluene). The minimum $T_{1}$ shifts to higher by blending similar to $T_{\min }$. Methylene and chloromethylene carbon show similar behavior. $T_{\text {min }}$ and the minimum $T_{1}$ are shown in Table II.

$T_{1}$ depends on molecular motion. ${ }^{7}$ The higher shifts of $T_{\min }$ show decrease of molecular motion. So it can be said that the molecular motion of $\mathrm{PECH}$ is inhibited by surrounding PMMA chains, and the molecular motion of the blend film cast from THF is influenced by PMMA

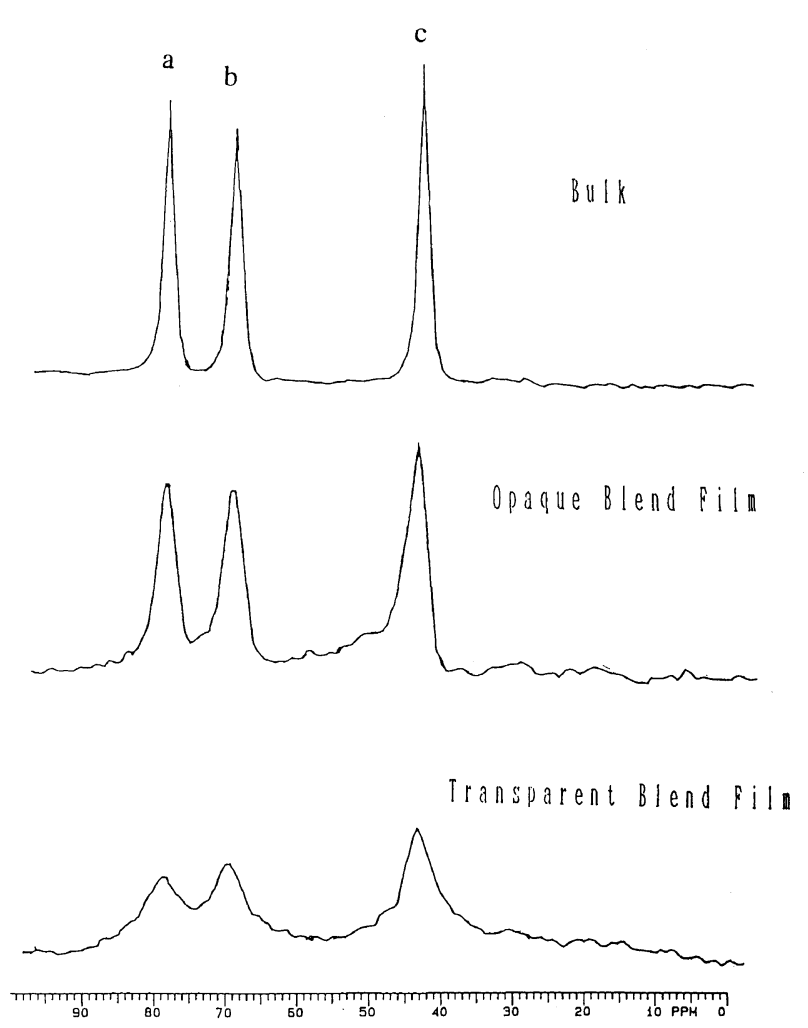

Figure 2. ${ }^{13} \mathrm{C} \mathrm{NMR}$ spectra of $\mathrm{PECH}$, peak (a) represents methine carbon, (b) methylene and (c) chloromethylene, pulse width is $25 \mu \mathrm{s}$ and transients are 256 .

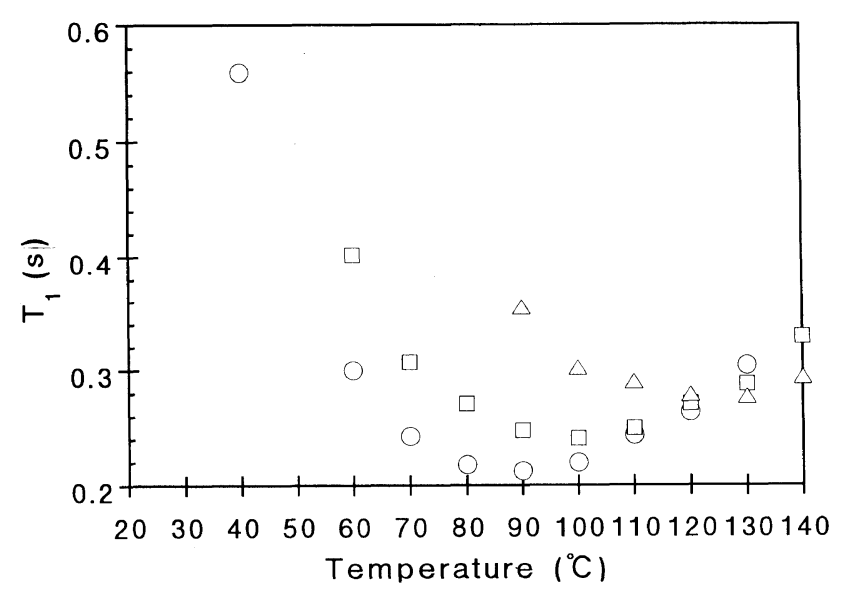

Figure 3. Temperature dependence of $T_{1}$ in PECH (methine carbon). $\bigcirc$, bulk; $\square$, blend film cast from THF (opaque); $\triangle$, blend film cast from toluene (transparent).

chain. But the molecular motion of $\mathrm{PECH}$ in the blend film cast from THF is not much inhibited by PMMA chains compared to those cast from toluene. So it may be safely said that there is a $\mathrm{PECH}$ rich phase in the blend film cast from THF. The higher shifts of minimum value of $T_{1}$ show increase of distribution of correlation time on molecular motion.

\section{Temperature Dependence of Spin-Spin Relaxation Time $\left(\mathrm{T}_{2}\right)$ in $\mathrm{PECH}$}

Figure 4 shows spin-spin relaxation decay of methine carbon in $\mathrm{PECH}$ at $140^{\circ} \mathrm{C}$. We can get $T_{2}$ by fitting the spin-spin relaxation decay curve to the eq 1 . 
Table II. Temperature $\left(T_{\min }\right)$ and $T_{1}$ where $T_{1}$ is minimal

\begin{tabular}{|c|c|c|c|c|c|c|}
\hline & \multicolumn{2}{|c|}{ Methine } & \multicolumn{2}{|c|}{ Methylene } & \multicolumn{2}{|c|}{ Chloromethylene } \\
\hline & $T_{\min } /{ }^{\circ} \mathrm{C}$ & $T_{1} \mathrm{minimum} / \mathrm{s}$ & $T_{\min } /{ }^{\circ} \mathrm{C}$ & $T_{1}$ minimum $/ \mathrm{s}$ & $T_{\min } /{ }^{\circ} \mathrm{C}$ & $T_{1} \operatorname{minimum} / \mathrm{s}$ \\
\hline Opaque blend cast from THF & 100 & 0.241 & 100 & 0.152 & 80 & 0.155 \\
\hline Transparent blend cast from toluene & 130 & 0.277 & 110 & 0.178 & 90 & 0.200 \\
\hline
\end{tabular}

$T_{\min }$ is the temperature where $T_{1}$ is minimal. $T_{1}$ minimum is the minimum of $T_{1}$.

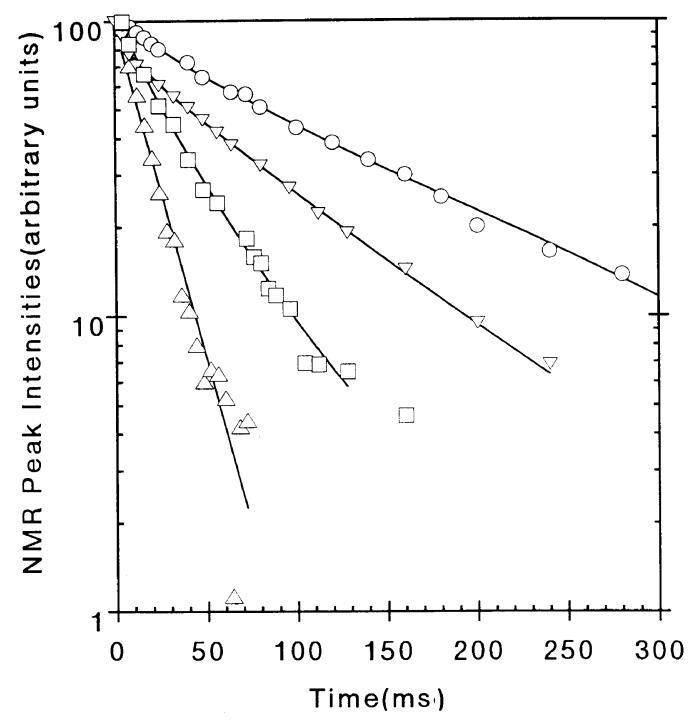

Figure 4. Spin-spin relaxation decay of methine carbon in $\mathrm{PECH}$ at $140^{\circ} \mathrm{C}$. $\bigcirc$, bulk; $\nabla$, blend film cast from $\mathrm{THF} / \mathrm{H}_{2} \mathrm{O}$ (opaque); $\square$, blend film cast from THF (opaque); $\triangle$, blend film cast from toluene (transparent). The lines, calculated distribution curve analysis.

$$
M(t)=M(0) \exp \left(-t / T_{2}\right)
$$

where $M(t)$ is signal intensity at delay time $=t, M(0)$ signal intensity, $t$ delay time and $T_{2} T_{2}$. In Figure 4, the decay curves cannot be represented by single relaxation times described with eq 1 and indicate that there exist multiple components with different $T_{2} . T_{2}$ in the opaque blend films are average values in the multiple relaxation times.

Temperature dependence of $T_{2}$ in methine carbon is shown in Figure 5. In Figure 5, $T_{2}$ in methine carbon increases with temperature. But $T_{2}$ of blend films is shorter than that of bulk. This shows that the molecular motion of PECH in blend film is inhibited by surrounding PMMA chains. $T_{2}$ of blend film cast from THF shows intermediate behavior of bulk and transparent blend film. It shows that the molecular motion of PECH in the opaque blend film is influenced by PMMA, but not so much as in the transparent blend film. These results are consistent with $T_{1}$. But the difference of temperature where $T_{2}$ shows same value is larger than the difference of $T_{\min }$ of $T_{1}$, which means $T_{2}$ is more sensitive to compatibility than $T_{1}$. Slow motion described by $T_{2}$ is more influenced by blending than the fast one described by $T_{1} . T_{2}$ of methylene carbon and chloromethylene carbon show similar behavior.

\section{Distribution Curve Analysis of $T_{2}$ and Characterization of Inhomogeneity}

We observed the spin-spin relaxation of opaque blend film cast from $\mathrm{THF} / \mathrm{H}_{2} \mathrm{O}$ as well as the other three

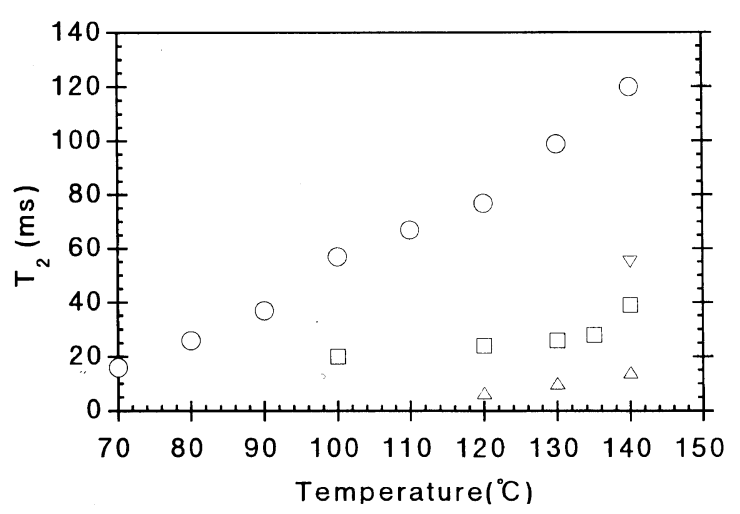

Figure 5. Temperature dependence of $T_{2}$ in $\mathrm{PECH}$ (methine carbon). $O$, bulk; $\nabla$, blend film cast from $\mathrm{THF} / \mathrm{H}_{2} \mathrm{O}$ (opaque); $\square$, blend film cast from THF (opaque); $\triangle$, blend film cast from toluene (transparent).

samples. This is because that $T_{2}$ is more sensitive to compatibility than $T_{1}$ and that $T_{2}$ is most sensitive at $140^{\circ} \mathrm{C}$ during our measurement.

The spin-spin relaxation decay in Figure 4 indicates multiple components with different $T_{2}$, but not the number of components.

When the observed signal consists of several $T_{2}$ components, the relaxation decay in Figure 5 can be represented by eq 2 .

$$
M(t)=\sum_{i=1}^{n} A i \exp \left(-t / T_{2 i}\right)
$$

where $t$ is delay time, $M(t)$ signal intensity at delay time $t, n$ number of components, $A i$ intensity of $i$-th component, $T_{2 i} T_{2}$ of $i$-th component.

But it is difficult to determine the number and $T_{2}$ of the components in opaque blend films.

So we got the distribution curves of $T_{2}$ from curve fitting by eq 2. $T_{2 i}$ values are predetermined and spread over the time range and fixed in eq 2 . We can get the best fitted $A i$ by non-linear least squares method. In this method, minimization of squared errors is made by varying the intensities of components $(A i)$ only. In the case of bulk, the estimated $A i$ values show zero except the $T_{2}$ range from $105 \mathrm{~ms}$ to $190 \mathrm{~ms}$ even though predetermined $T_{2 i}$ values are spread over the time range wider. So we determined the range of $T_{2 i}$ values in bulk from $105 \mathrm{~ms}$ to $190 \mathrm{~ms}$. The squared errors decrease as $n$ increases until 12. We determined the number of components $(n)$ to 12 . For blend films, we determined the range of $T_{2 i}$ values and $\mathrm{n}$ like the case of bulk.

The results of the distribution curve analysis of Figure 4 are shown in Figure 6. In Figure 6, the bulk and blend film cast from toluene show sharp distributions. This indicates that bulk and transparent blend film are 


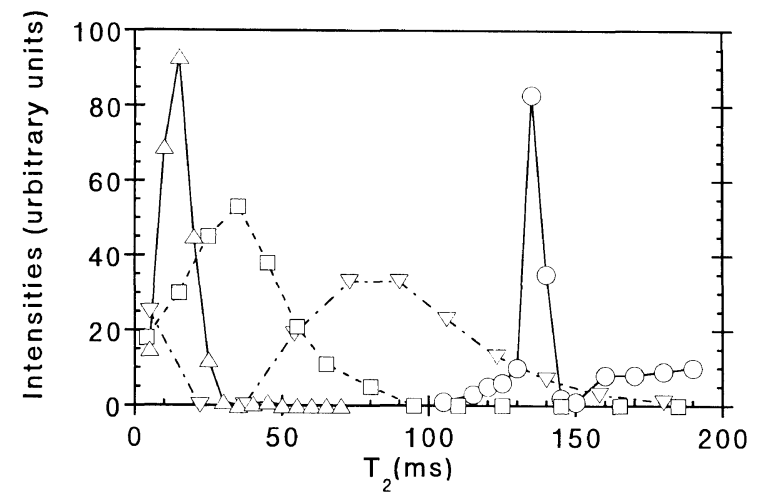

Figure 6. Distribution curves of $T_{2}$ in PECH (methine carbon). markings: calculated intensities $(A i), \bigcirc$, bulk; $\nabla$, blend film cast from $\mathrm{THF} / \mathrm{H}_{2} \mathrm{O}$ (opaque); $\square$, blend film cast from THF (opaque); $\triangle$, blend film cast from toluene (transparent). The lines, distribution lines guessed from $A i$.

homogeneous.

But in the case of the opaque blend film cast from $\mathrm{THF}$, the components are distributed over a wide range of $T_{2}$; from $5 \mathrm{~ms}$ to $100 \mathrm{~ms}$. In the distribution curve, the $T_{2}$ range from $5 \mathrm{~ms}$ to $20 \mathrm{~ms}$ is equal to that of the transparent blend film. So the opaque blend film cast from THF contains the PECH chains where the molecular motion described by $T_{2}$ is equal to that of the transparent blend film. In the distribution curve, $T_{2}$ range from $20 \mathrm{~ms}$ to $100 \mathrm{~ms}$ indicates the existence of the $\mathrm{PECH}$ polymer chains as intermediate molecular mobilities between transparent blend film and bulk. There are no intensities in the range from $120 \mathrm{~ms}$ to $150 \mathrm{~ms}$. The range from $120 \mathrm{~ms}$ to $150 \mathrm{~ms}$ is equal to that of bulk. This shows there is no $\mathrm{PECH}$ polymer chains having equal molecular motion to bulk.

The wide range distribution of $T_{2}$ indicates the existence of polymer chains having different mobilities, and the mobilities of molecular chains have wide distribution. That the distribution curve is wide and continuous suggests a gradient in molecular motions of $\mathrm{PECH}$ described by $T_{2}$.

From these results, we consider the existence of gradient in local concentration of PECH and PMMA in the opaque blend film cast from THF. It is considered that the existence of PECH polymer chains in the local concentration with PMMA is equal to that of the transparent blend film, which has the component distribution over $T_{2}$ range from $5 \mathrm{~ms}$ to $20 \mathrm{~ms}$. From the components distributed over the $T_{2}$ range from $20 \mathrm{~ms}$ to $100 \mathrm{~ms}$, it is suggested that the blend film has the $\mathrm{PECH}$ chains where the local concentration of $\mathrm{PECH}$ is higher than in transparent blend.

In the case of the opaque blend film cast from THF/ $\mathrm{H}_{2} \mathrm{O}$ solution, the main part of the distribution curve is the range between $35 \mathrm{~ms}$ and $150 \mathrm{~ms}$ of $T_{2}$. It is wider than that of the film cast from THF solution. So there may exist $\mathrm{PECH}$ polymer chains with intermediate molecular mobilities between the blend film cast from THF solution and bulk. Thus the local concentration of $\mathrm{PECH}$ rich polymer chains is higher in this film cast from THF solution, and the distribution of local concentration is wider. Therefore, PECH and PMMA are much separated by water in the cast solution.

The results of the distribution curve analysis from

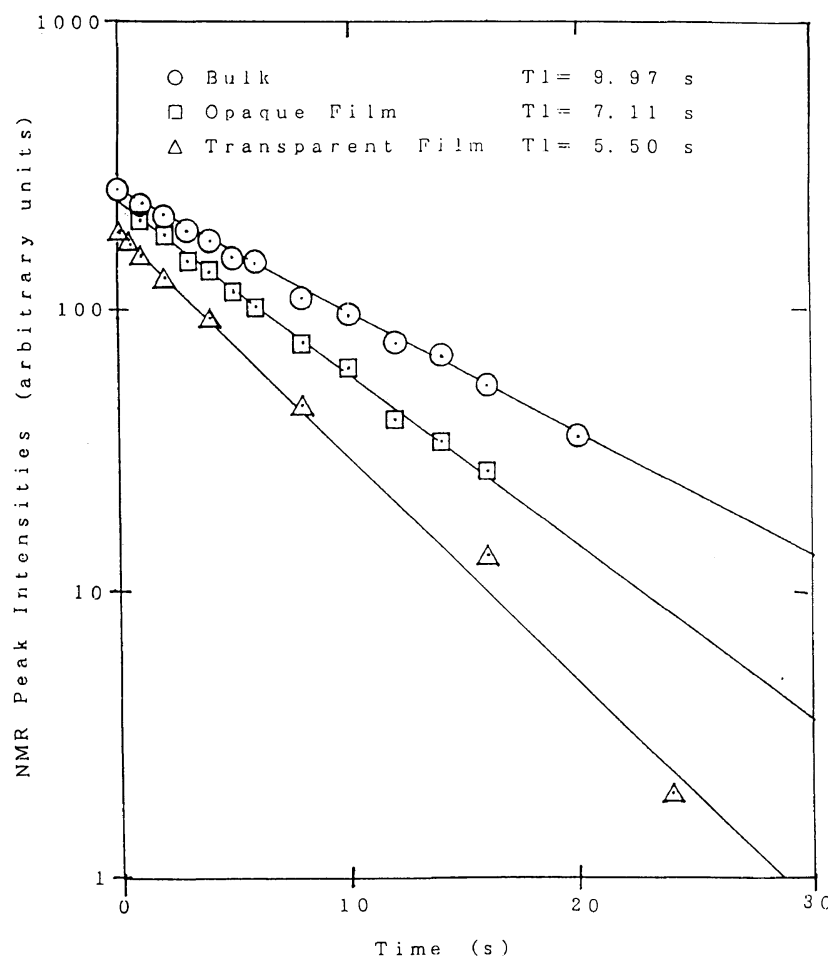

Figure 7. Spin-lattice relaxation decay of methoxy carbon in PMMA O, bulk; $\square$, blend film cast from THF (opaque); $\triangle$, blend film cast from toluene (transparent). The lines are calculated by single-exponential decay.

spin-spin relaxation of methylene carbon and of chloromethylene carbon in PECH indicate the similar behavior with those of methine carbon.

In the case of the opaque blend film cast from $\mathrm{THF} /$ $\mathrm{H}_{2} \mathrm{O}$, the distribution curve of methine carbon has a small component in the range of about $5 \mathrm{~ms}$. The component is separated from another component distributed over the range between $35 \mathrm{~ms}$ and $150 \mathrm{~ms}$. So there may be a separated phase in this film. But there are no such components in the distribution curves of methylene carbon and chloromethylene carbon. So it is considered that only the molecular motion of methine carbon is much decreased in this phase.

Spin-Lattice Relaxation of PMMA at Room Temperature

Spin-lattice relaxation of methoxy carbon in PMMA is shown in Figure 7, where we got spin-lattice relaxation time $\left(T_{1}\right) . T_{1}$ of transparent blend film is shorter than that of PMMA bulk. $T_{1}$ of the opaque blend film cast from THF is intermediate that of transparent blend film and PMMA bulk. We consider that the molecular motion of PMMA polymer chains in the blend films increases by surrounding PECH polymer chains. The increase of molecular mobility of PMMA chains depends on the compatibility of PMMA and PECH. This agrees with the result of $\mathrm{PECH}$. But we cannot discuss molecular motion of PMMA chains with only the present experimental results. We need temperature dependence of $T_{1}$ etc. for discussion about molecular motions of PMMA

\section{CONCLUSIONS}

(1) From the line shape and temperature dependence 
of $T_{1}$ and of $T_{2}$, molecular motion of PECH chains in blend film PECH/PMMA are inhibited by surrounding PMMA chains and the decrease in molecular motion depends on the compatibility.

(2) The slow motion described by $T_{2}$ is more influenced by blending than the fast one described by $T_{1}$.

(3) Analysis of the distribution of $T_{2}$ is useful for investigating the inhomogeneity of the blend film.

(4) The transparent blend film cast from toluene solution and PECH bulk have the sharp distribution of molecular motion in $\mathrm{PECH}$, and are homogeneous in molecular motion.

(5) In opaque blend film cast from THF solution, there are $\mathrm{PECH}$ polymer chains with different mobilities, and wide and continuous distribution.

(6) The width of distribution range of $T_{2}$ depends on the cast solutions and wide distribution in the opaque blend films may indicate a gradient in local concentration of PECH. The distribution of local concentration becomes wider by water in cast solution.

(7) $T_{1}$ of methoxy carbon in PMMA shows change of molecular motion of PMMA chains by blending with $\mathrm{PECH}$, and this agrees with the result of PECH.

\section{REFERENCES}

1. D. R. Paul and S. Newman, "Polymer Blends," Vols. I and II, Academic Press, New York, N.Y., 1978.

2. J. R. Fried, F. E. Karasz, and W. J. MacKnight, Macromolecules, 11, 150 (1978).

3. K. Naito, G. E. Johnson, D. L. Allara, and T. K. Kwei, Macromolecules, 11, 1260 (1978).

4. S. Akiyama, K. Komatsu, and R. Kaneko, Polym. J., 7, 172
(1975).

5. M. Bank, J. Loffingwell, and C. Thies, Macromolecules, 4, 43 (1971).

6. T. C. Farrar and E. D. Becker, "Pulse and Fourier Transform NMR," Academic Press, New York, N.Y., 1971.

7. T. Nishi, T. T. Warg, and T. K. Kwei, Macromolecules, 7, 667 (1974).

8. D. C. Douglass and V. J. McBrierty, Macromolecules, 11, 766 (1978).

9. V. J. McBrierty and D. C. Douglass, Macromolecules, 11, 1265 (1978).

10. R. A. Assink and G. L. Wilkes, Polym. Eng. Sci., 17, 606 (1977).

11. A. Tsutsumi and C. Chachaty, Macromolecules, 12, 429 (1979).

12. E. D. Becker, "High Resolution NMR Theory and Chemical Applications," Academic Press, New York, N.Y., 1980.

13. R. Voelkel, Angew. Chem. Int. Ed. Engl., 27, 1468 (1988).

14. B. Gabrys, F. Horii, and R. Kitamaru, Macromolecules, 20, 175 (1987).

15. R. Kitamaru, F. Horii, and K. Murayama, Macromolecules, 19, 636 (1986)

16. F. Horii, A. Hirai, and R. Kitamaru, Macromolecules, 20, 2117 (1987).

17. A. Kaji, Y. Ohta, H. Yasuda, and M. Murano, Polym. J., 22, 455 (1990).

18. X. Zang, K. Takegoshi, and K. Hikichi, Macromolecules, 25, 2336 (1992)

19. J .F. Parmer, L. C. Dickinson, J. C. W. Chien, and R. S. Porter, Macromolecules, 22, 1078 (1989).

20. P. Carabatti, P. Neuenschwander, and R. R. Ernst, Macromolecules, 19, 1889 (1985).

21. P. Carabatti, P. Neuenschwander, and R. R. Ernst, Macromolecules, 18, 119 (1985).

22. F. Horii, A. Hirai, K. Murayama, R. Kitamaru, and T. Suzuki, Macromolecules, 16, 273 (1983).

23. C. Anderson and F. Rodriguez, Polym. Mater. Sci. Eng., 51, 604 (1984).

24. A. C. Fernandes, J. W. Barlow, and D. R. Paul, J. Appl. Polym. Sci., 32, 5481 (1986). 